\title{
Implementation of Fault Diagnosis in a Dynamic Process
}

\author{
C. ANGELI and A. CHATZINIKOLAOU
}

\begin{abstract}
The increasing complexity of industrial systems leads to increased requirements concerning diagnosis capabilities. An intelligent process monitoring and fault diagnosis environment has been developed by interfacing a data acquisition and control software with a knowledge base system in an effective way. The abrupt changes of important system's variables are continually captured, analysed and compared with the relevant estimated values and the results are used for the final knowledge based diagnostic conclusions. The presentation of the implementation and developing of the intelligent monitoring process is the overall goal of this paper.
\end{abstract}

Keywords - Modelling, Simulation, Fault diagnosis, Dynamic Process.

\section{Introduction}

A large diversity of advanced methods for automated fault detection and isolation exists based on the fault diagnosis principle. Faults in systems are usually diagnosed using analytical redundancy by comparing the measured and the corresponding estimated outputs of the systems behaviour.

\section{ANGELI}

Piraeus University of Applied Sciences

Laboratory of Applied Mathematics and Artificial

Intelligence

P. Ralli \& Thivon 250

GR-171 21 Athens, Greece

\section{A. CHATZINIKOLAOU}

Laboratory of Applied Mathematics and Artificial

Intelligence

S. Patsi 62

GR-118 55 Athens, Greece
The diagnostic procedure is composed of three stages: residual generation, residual evaluation and finally decision making.

In the stage of symptom generation one of the main problems is the coping with the systems dynamics. Model-based fault diagnosis for dynamical systems is a well established approach and a lot of research work has been published over the last years such as [1], [2], [3], [4], [5], [6], [7].

Difficulties with model based fault detection methods arise from the fact that the accuracy of the measurements needed to calculate the evolution of faults should be of high quality. In addition, use of model-based methods may require assumptions about the process that are not valid as the degree of influence of noise and disturbances to the fault detection process.

The difficulty by the search for defective components in case of a malfunction in the fluid power systems due to their inaccessibility and the high working pressures has driven to some attempts to implement automated systems in this domain for diagnostic purposes. Recent research work on developing diagnostic and monitoring methods for hydraulic actuators includes [8], [9], [10] where a detailed causes of malfunction of the overall conditions in the system is concluded.

This paper refers to the development of a component fault diagnostic method under dynamic conditions. The fault detection process is implemented using a data acquisition and control software for the monitoring process and a knowledge-based framework for the final diagnosis of faults. The data acquisition software permits the qualification of the 
numerical measured data and as consequence makes possible the on-line interaction with the symbolic information of the knowledge base.

The paper is organized as follows: Section 2 presents the implementation of the monitoring process of important variables of the system. Section 3 focuses on the data acquisition subsystem, while Section 4 presents the fault diagnosis system the justification of the results. Finally, Section 5 includes concluding remarks. Recent research work in diagnostic procedures for fluid power systems includes [11], [12], [13].

\section{Implementation Process Monitoring}

The main electric devices of the physical system are the electric motor, the proportional pressure valve for the control of the main pressure, the proportional 4-way valve for the control of the motor speed, the pressure transducers for the measurement of the pressure and the logic levels for the pressure switch, the pressure switch for the indication of the

fluid contamination and the oil level switch.

Electrical input signals are applied to the electric motor, to the proportional pressure valve and to the proportional 4-way valve. Electrical output signals are produced by the pressure transducers, the filter indicator and the oil level indicator.

The measurement of pressure is performed by pressure transducers and the measurement of the angular velocity of the hydraulic motor is performed by an incremental rotary encoder.

The electronic interface consists mainly of a multiple power supply module, a module with the relays for the input and output signals and the amplifier cards for the control of the proportional valves.

The layout of the rear panel of the electronic interface is illustrated in Figure 1.

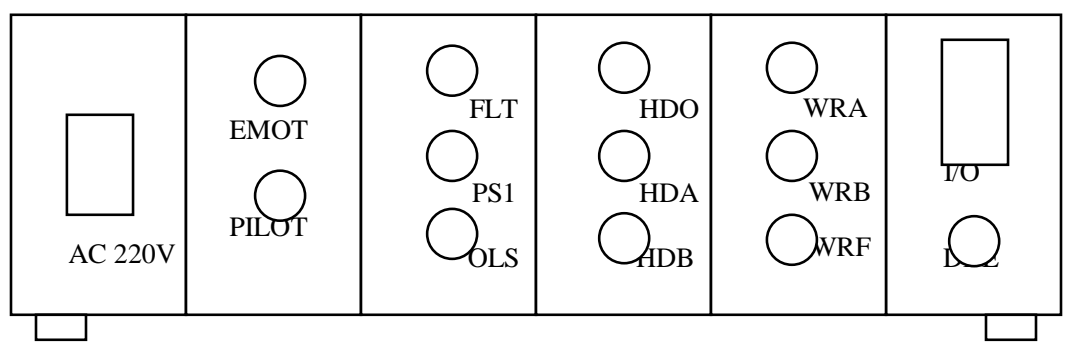

Figure 1. Rear panel of the electronic interface

This panel includes the connections to the electric motor (EMOT), the electric pilot of the main relief valve (PILOT), the filter (FLT), the pressure switch (PS1), the oil level switch (OLS), the pressure transducers (HDO, HDA, HDB), the solenoids and the feedback of the proportional 4-way valve (WRA,WRB, WRF), the solenoid of the proportional pressure valve (DBE) and the I/O card.

\section{The data acquisition sub-system}

The focus of the data acquisition system for this application is the measurement of the pressures $\mathrm{p}_{\mathrm{a}}$, $\mathrm{p}_{\mathrm{b}}$ and the angular velocity $\omega$ as well as the monitoring of the digital signals that indicate the correct operation of the electric motor and other electrical devices of the system as filter, oil level switch e.t.c.

The data acquisition system runs in parallel to the actual system and acquires data for the angular velocity and the pressures while the speed is changing. Figure 2 shows the data acquisition process for the pressures and the motor speed. 
Proc. of the Fourth International Conference on Advances in Computing, Electronics and Communication - ACEC 2016. Copyright (C) Institute of Research Engineers and Doctors. All rights reserved.

ISBN: 978-1-63248-113-9 doi: 10.15224/ 978-1-63248-113-9-54

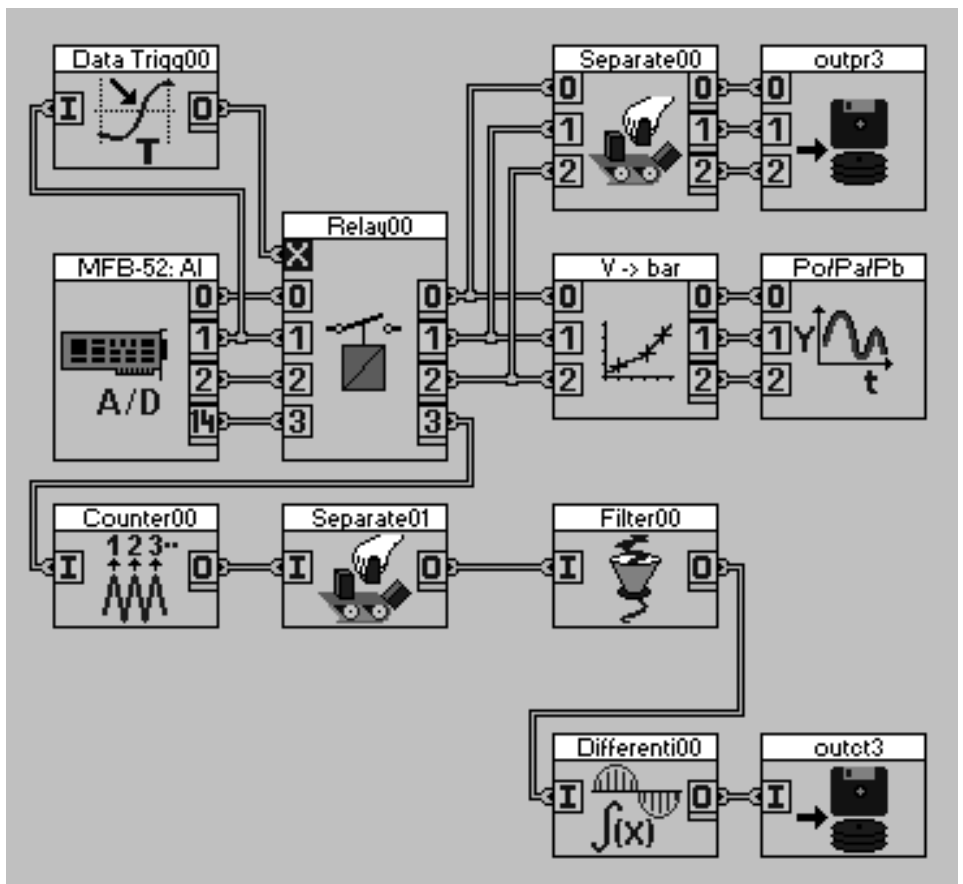

Figure 2. The data acquisition process

The digital data acquisition process is performed by

the part of the data acquisition system shown in Figure 3.

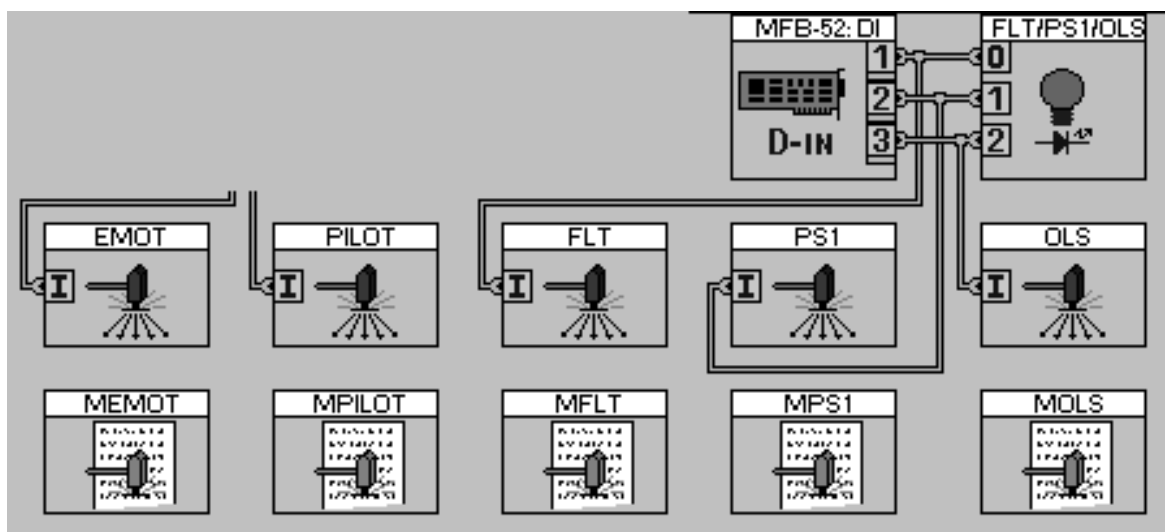

Figure 3. The handling of digital signals 
In the worksheet of Figure 3 digital module of the I/O card (MFB-52: DI:) receives the digital input signals with the corresponding $\mathrm{I} / \mathrm{O}$ of the installed data acquisition hardware. For each activated digital input channel the module provides one output, through which the acquired values will be sent to other modules in the worksheet. The three channels are associated with the filter contamination indicator (FLT), pressure switch (PS1) and oil level indicator (OLS). Through these channels the digital data acquisition is performed. The FLT/PS1/OLS: is a "triple status display" module for the status indication of the filter contamination (FLT), the system pressure (PS1), and the oil level (OLS).

The module EMOT refers to the status of the electrical motor, is activated from the corresponding digital signal of MFB52:DI module and acts on the corresponding message module MEMOT which indicates if the electric motor is running or not (OK or not $\mathrm{OK}$ ) and writes this information to a text file for further processing by the expert system.

Similarly the module PILOT referring to the electrical pilot of the pressure valve writes a message to the module MPILOT that indicates if the command for the energising of the electrical pilot of the main relief valve is given. Similar function have the modules FLT and MFLT for the state of the filter, PS1 and MPS1 for the pressure switch and OLS and MOLS for the oil level.

The effects on the monitoring system of the operation of the worksheet of figure 4 is presented in Figure 4 for the case that the filter is clean the pressure switch is energised and the oil level is too low.

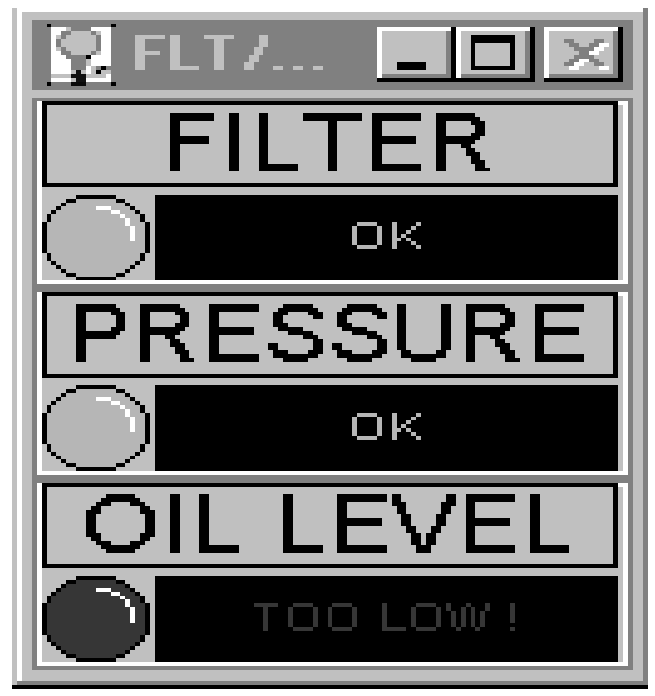

Figure 4. Display of the digital signals

\section{The Fault Diagnosis process}

Faults are diagnosed by determining the changes in variables that have significant contribution to the diagnostic process and by relating these variables to specific process equipment faults. This information is used by the expert system as input together with the text file information coming from the data acquisition process and experiential knowledge to determine the faulty component. 
In following Figure 5 measured and calculated data for the pressure values $\mathrm{p}_{\mathrm{a}}$ are simultaneously presented for the fault free case.

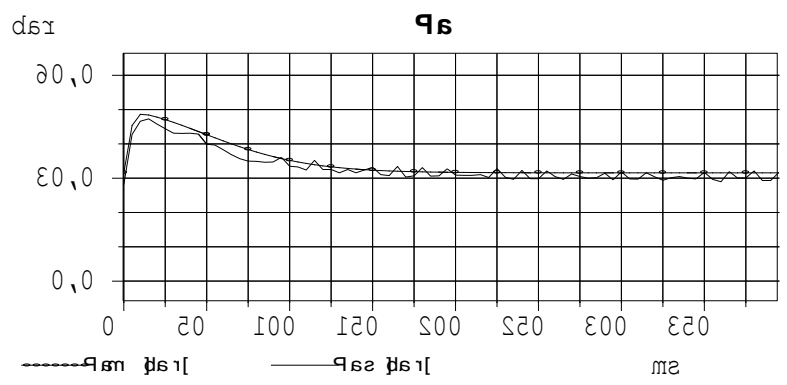

Figure 5. Measured and calculated pressure values

The measured variable values and the values of the logic signals are transformed to qualitative information which can be used by the knowledge base of the system for the final conclusion. These symbolic information can be passed in the structure of the knowledge representation scheme and trigger specific set of rules that are organised under the structure of the topic.

Faults are classified in different categories depending on their influence on the performance of the total system.

Digital inputs contain information that refers to primary faults. The corresponding topics are checked at the beginning of every search. In the case that a fault is not established in these branches of the fault tree, the search is continued in other branches with more depth by checking the parameters the measured values.

\section{Conclusion}

Components, sensors and actuators in physical systems are often subjected to unexpected and unpermitted deviations from acceptable conditions for many reasons that can cause loss of the overall performance of the system and may lead to unacceptable economic loss.

In this paper the monitoring and the fault diagnosis of a dynamic process was presented by a combination of suitable environments and tools. This method enables quick and reliable detection of process abnormalities and trigger fault diagnostic activities. The knowledge based system provides the suitable environment for the fault diagnosis by combining on-line signal information and stored knowledge.

The main benefits gained of this interaction lie on the offered higher degree of safety and reliability for industrial systems by continuous monitoring of changes. 


\section{References}

[1] R. Patton, P. Frank and R.Clark, "Issues in Fault Diagnosis For Dynamic Systems", Springer-Verlag, 2000.

[2] R. Isermann, "Model-based Fault Detection and Diagnosis: Status and Applications". Annual Reviews in Control, 29, 2005, pp. 71-85.

[3] S. Narasimhan, and G. Biswas, "Model-based diagnosis of hybrid systems", IEEE Transactions on Systems, Man and Cybernetics, A Syst. Humans, 2007, Vol. 37, No. 3, pp. 348-361.

[4] S. Ghoshal, and S. Samanta, "Multiple Fault Isolation to a Servo-Valve Controlled Motor Transmission System", In Proceedings $7^{\text {th }}$ IFAC Safeprocess 09, 2009, Barcelona, Spain.

[5] R. Isermann, Fault diagnosis applications: Model-based condition monitoring: Actuators, Drives, Machinery, Plants, Sensors and Fault tolerant Systems, Springer, Berlin, 2011.

[6] J. Korbicz, J. Koscielny., Z.. Kowalczuk and W. Cholewa "Fault Diagnosis: Models, Artificial Intelligence, Applications", Springer Verlag, Berlin, 2004.

[7] C. Angeli, "On-line Expert Systems for Fault Diagnosis in Technical Processes”. Expert Systems, Vol. 25, No2, 2008, pp. $115-132$.

[8] D.. Krokavec and A. Filasova, "An Approach to reconstruction of actuator faults for a class of nonlinear systems", In Proceedings $8^{\text {th }}$ IFAC Safeprocess 12, Mexico City, Mexico, 2012, pp. 216-221.

[9] L Marton. and D. Ossmann, "Energetic Approach for Control Surface Disconnection Fault Detection in hydraulic aircraft actuators, In Proceedings $8^{\text {th }}$ IFAC Safeprocess 12, Mexico City, Mexico, 2012, pp. 1149-1154.

[10] M. Muenchhof and S. Clever, "Neural-Net Based Fault Diagnosis for Hydraulic Servo Axes", In Proceedings $7^{\text {th }}$ IFAC Safeprocess 09, Barcelona, Spain, 2009.

[11] C.Angeli and A. Chatzinikolaou "Troubleshooting in Hydraulic Systems using knowledge-based Methods", International Journal of Engineering Simulation, Vol. 6, Nr.1, 2005, pp. 24-29.

[12] M. Muenchhof and S. Clever, "Neural-Net Based Fault Diagnosis for Hydraulic Servo Axes", In Proceedings $7^{\text {th }}$ IFAC Safeprocess 09, Barcelona, Spain, 2009.

[13] Leonardo, D., Kleinmann, S., Koller-Hodac, A., Stetter, R.: Simulation, monitoring and diagnosis methods for a hydraulic pump application. In Proceedings of Case 2011, Trieste, August 24-27, 2011. 